\title{
Video Surveillance using Distance Maps
}

\author{
Theo E. Schouten ${ }^{a}$, Harco C. Kuppens ${ }^{a}$, and Egon L. van den Broek ${ }^{b, c}$ \\ ${ }^{a}$ Nijmegen Institute for Computing and Information Science, Radboud University Nijmegen \\ P.O. Box 9010, 6500 KL Nijmegen, The Netherlands \\ \{T.Schouten, H.Kuppens\}@cs.ru.n] \\ ${ }^{b}$ Faculty of Behavioral Sciences, University Twente \\ P.O. box 217, 7500 AE Enschede, The Netherlands \\ e.l.vandenbroek@utwente.nl \\ http://users.gw . utwente.nl/BroekEL/ \\ ${ }^{c}$ Department of Artificial Intelligence, Faculty of Sciences, Vrije Universiteit Amsterdam \\ De Boelelaan 1081a, 1081 HV Amsterdam, The Netherlands \\ egon@few.vu.nl \\ http://www.few.vu.nl/ egon/
}

\begin{abstract}
Human vigilance is limited; hence, automatic motion and distance detection is one of the central issues in video surveillance. Hereby, many aspects are of importance, this paper specially addresses: efficiency, achieving real-time performance, accuracy, and robustness against various noise factors. To obtain fully controlled test environments, an artificial development center for robot navigation is introduced in which several parameters can be set (e.g., number of objects, trajectories and type and amount of noise). In the videos, for each following frame, movement of stationary objects is detected and pixels of moving objects are located from which moving objects are identified in a robust way. An Exact Euclidean Distance Map $\left(\mathrm{E}^{2} \mathrm{DM}\right)$ is utilized to determine accurately the distances between moving and stationary objects. Together with the determined distances between moving objects and the detected movement of stationary objects, this provides the input for detecting unwanted situations in the scene. Further, each intelligent object (e.g., a robot), is provided with its $\mathrm{E}^{2} \mathrm{DM}$, allowing the object to plan its course of action. Timing results are specified for each program block of the processing chain for 20 different setups. So, the current paper presents extensive, experimentally controlled research on real-time, accurate, and robust motion detection for video surveillance, using $\mathrm{E}^{2} \mathrm{DMs}$, which makes it a unique approach.
\end{abstract}

\section{INTRODUCTION}

In video processing, it is often needed to detect motion; i.e., it is required to extract information about the relative positions of stationary and moving objects. The resulting distances between the objects can, for example, be used for surveillance purposes. Unwanted or possible dangerous distances can be automatically detected (e.g., too small or too large), in order to take appropriate actions. The automation of this process is of the utmost importance since the vigilance of humans is limited. The ability of humans to hold attention and to react to rarely occurring events, is extremely demanding and, consequently, prone to error due to lapses in human attention ${ }^{1}$. These actions might include intervention by a (human) supervisor watching the video or sending relevant distance information to objects (or persons) that have capabilities for changing the course of their actions ${ }^{1-3}$.

Amer and Regazzoni ${ }^{3}$ state that video-based surveillance is one of the fastest growing sectors in the security market due to the high amount of useful information that can be extracted from a video sequence. They present a generic block diagram of a video processing module for video surveillance and identify a list of nine fundamental issues and challenges: interpretation, generality, automation, efficiency, robustness, trade off (accuracy versus efficiency), performance evaluation, multiple camera processing and data fusion, and feature selection and integration. This broad range of issues illustrates the complexity of extracting the useful information from the large amount of data present in a video sequence. In this paper, we concentrate on two issues as identified by Amer and Regazzoni ${ }^{3}$, namely: efficiency, which is the core focus, and robustness, considered as essential and, hence, tested as well.

We choose for an operational environment where a single camera provides a top view of a scene with moving and stationary objects. The conversion of the gray and color images of the camera to binary (i.e., black-andwhite) images, which are used for further processing, will not be discussed. However, we do take into account 
that this conversion process is not able to handle all the challenges posed by changing illumination conditions, shadows and video noise correctly. We model the conversion and its imperfections by an extension of our virtual, dynamic robot navigation environment ${ }^{4}$.

The calculation of distances is based on the Fast Exact Euclidean Distance (FEED) transformation developed by Schouten and Van den Broek ${ }^{5}$. This Euclidean Distance Transformation converts a binary image to another image of the same size, such that each pixel has a value equal to the exact Euclidean distance to the nearest object pixel, where most other algorithms approximate the true Euclidean distance ${ }^{6,7}$. The new image is called the exact Euclidean Distance Map $\left(\mathrm{E}^{2} \mathrm{DM}\right)$ of the old image. With the calculation of the $\mathrm{E}^{2} \mathrm{DM}$ for stationary objects, the distance of a moving object to the stationary object can be calculated by taking the minimum of the distances in this $\mathrm{E}^{2} \mathrm{DM}$ at the locations of the border pixels of the moving object.

As shown by Schouten, Kuppens, and Van den Broek ${ }^{4}$, the $\mathrm{E}^{2} \mathrm{DM}$ of images containing stationary and moving objects can be obtained very fast, using the FEED transformation. Here, very fast denotes that the $\mathrm{E}^{2} \mathrm{DM}$ s of a sequence of more than a few images can be obtained faster than city-block ${ }^{8}$ or 3,4 chamfer $^{9}$ distance maps. To achieve the latter, the $\mathrm{E}^{2} \mathrm{DMs}$ for the stationary objects $\left(E^{2} D M_{\text {stationary }}\right)$ and for the moving object $\left(E^{2} D M_{\text {moving }}\right)$ are calculated separately and combined, using the minimum operator, to obtain the distance map for the total image:

$$
E^{2} D M_{\text {stationary+moving }}(p)=\min \left\{E^{2} D M_{\text {stationary }}(p), E^{2} D M_{\text {moving }}(p)\right\}
$$

This $\mathrm{E}^{2} \mathrm{DM}$ is used to provide each object (e.g., a robot) with processing capabilities, with a map of objects and free space in its environment. The robot can use information obtained from the $\mathrm{E}^{2} \mathrm{DM}$ to achieve its goal; e.g., path planning ${ }^{10}$.

The processing chain developed in this paper consists of two phases:

1. The initialization phase: A number of sequential images are used to determine the stationary pixels. At the end of it, $E^{2} D M_{\text {stationary }}$ is calculated.

2. The processing phase: For each next image it is verified whether or not the stationary pixels have changed substantially. If they have done so, the initialization is entered again. Otherwise, the moving pixels are identified, from which the moving objects are determined in a robust way. Next, distances from each moving object to the fixed and the other moving objects are calculated and presented to the (human) supervisor. Finally, in case an object with processing capabilities is present (for the purposes of this paper we simply assume that one of the objects has this capability), its $\mathrm{E}^{2} \mathrm{DM}$ to the stationary and the other moving objects is calculated.

In the next section, the operational environment is described. Three compounds can be distinguished: 1) the creation of the robot navigation environments, 2) the method of modeling imperfections (noise), and 3) the specification of hardware and software parameters. In Section 3, the principle of Fast Exact Euclidean Distance (FEED) maps is explained and some (further) improvements are specified. Section 4 describes the processing chain for real-time and exact motion detection. In this processing chain, two phases can be distinguished: the initialization phase and the processing phase. Obtained execution times for the most demanding video sequence are given and the design guidelines used for obtaining the fast execution times are explained. The next Section describes the programming blocks in details, including visualization of the (intermediate) results. The overall timing results are provided for the various settings, determined by: two image sizes and five levels of noise on two platforms. We end the paper with a discussion (Section 6) that raps up the current research, describes follow up research, and draws some final conclusions.

\section{OPERATIONAL ENVIRONMENT}

As stated in Section 1, we choose for an operational environment where a single camera provides a top view of a scene with moving and stationary objects. In order to experiment with such environments, we have developed a virtual, dynamic robot navigation environment ${ }^{4}$. It generates a binary video stream by animation using Macromedia ${ }^{\circledR}$ Flash; see also ${ }^{4}$. This provides us the means to record video sequences in a fully controlled environment. Moreover, using this animation system has the following advantages: 
- Moving objects can be separated from the background, using layers.

- The creation of moving objects is easy: Drawing a line in a layer. Given a number of frames, Flash will generate the different frames with the object shifted along the path. In addition, rotation of the object can be specified.

- Animations can be changed easily since Flash uses vector graphics.

- The color map and with that the object indexation is preserved with the export from animation to gif images. Most animation programs interpolate color values at edges of objects, with the export of bitmap images. This results in a loss of object indexation.

To model the imperfections of the conversion from real camera images to binary images, noise was added to the output of the robot navigation environment, using three transformations:

- Each border pixel of an object was with a probability of $p_{1} \%$ changed into a background pixel. Here, a border pixel is defined as an object pixel with at least one of its 4 connected neighbors in the background ${ }^{5}$.

- Randomly chosen background pixels that lay next to a border pixel were changed into an object pixel, with a probability of $p_{2} \%$.

- Each pixel was changed into its inverse, with a probability of $p_{3} \%$.

For the current research, two video sequences were generated:

1. A sequence of 60 frames of $320 \times 240$ pixels with two moving objects being present during the full sequence. One of the moving objects collides once with a fixed object and displaces it.

2. A sequence of 120 frames of $640 \times 480$ with one permanently moving object present and up to two additional objects that randomly appear and disappear from the scene. Once during the sequence, one of the stationary objects displaces itself and once, a moving object bounces off a stationary object, which is displaced and then returns to its original position.

To each generated image sequence five different $p_{1} \%-p_{2} \%-p_{3} \%$ noise patterns were applied, ranging from 10\%-10\%-1\% to 50\%-50\%-5\%. Thus in total ten sequences with a total number of 900 frames were used for this paper. To provide an impression, two of the images are shown in Figure 1. Note that the large image looks "darker" because it is printed at the same scale as the small image.

Two execution platforms were use for developing and testing the algorithms. The first, further referred to as AMD, was a desktop PC with an AMD Athlon XP ${ }^{\circledR} 1666 \mathrm{MHz}$ processor, having $64 \mathrm{kB}$ L1 cache, 256kB L2 cache, and $512 \mathrm{MB}$ memory. The second, further referred to as INTEL, was a DELL Inspiron ${ }^{\circledR} 510 \mathrm{~m}$ laptop. It had an Intel Pentium ${ }^{\circledR} \mathrm{M} 725$ processor with a clock of $1600 \mathrm{MHz}$, no L1 cache, 2MB L2 cache, and 512MB memory. On both platforms, the Microsoft ${ }^{\circledR}$ Visual $\mathrm{C}++6.0$ programming environment was used in the standard release setting. Please note that no effort was spend on reducing the execution time by varying compiler optimization parameters, by using pointers instead of indices, or by exploiting the particular characteristics of one of the machines.

Timing measurement were performed by calling the Visual $\mathrm{C}++\operatorname{clock}()$ routine before and after a repeated number of executions of (parts) of programs on all images of a sequence. As the clock() routine returns elapsed time with a granularity of ten milliseconds, the number of repetitions is set such that the total elapsed time is more than one second. The error on a single elapsed time measurement is than less than $2 \%$, provided that the computer was not busy with other tasks like processing interrupts. This was guarded against by switching off unneeded sources of interrupts (e.g., networks) and by repeating the measurements a number of times and taking the minimum of these measurements.

All frames of a sequence were read in memory and consequently, were directly available to the developed programs. The measured times reported in this paper include the times needed to generate the images used for output to the (human) supervisor or to intelligent objects. The time needed to actually display these images or to communicate them is not reported in this paper. 


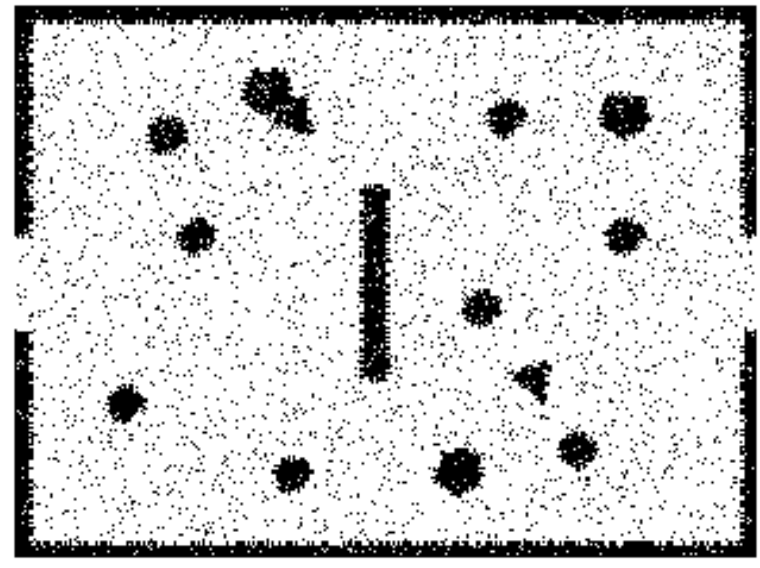

(a)

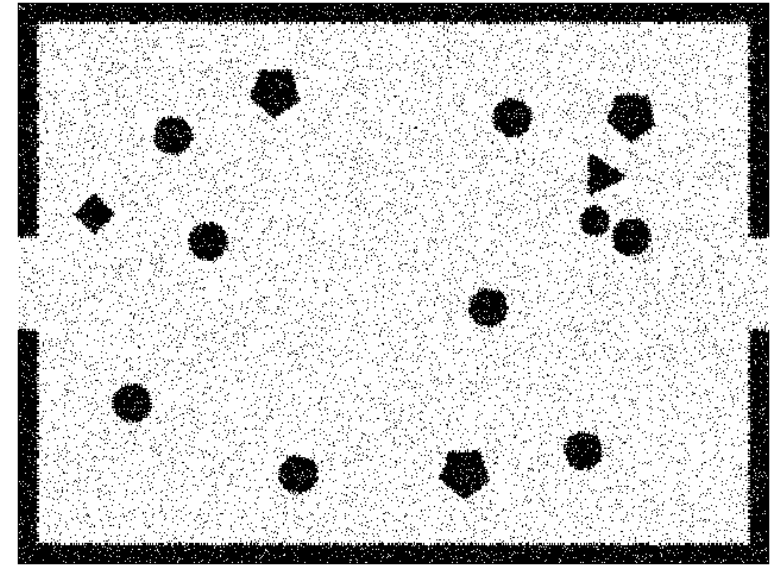

(b)

Figure 1. Example input images: (a) has size $320 \times 240$; the two triangles are the moving objects and the upper one just collides with and will move a stationary object. (b) has size $640 \times 480$ and three moving objects: (i) the triangle, (ii) the small circle below the triangle, and (iii) the square. Both images are generated with a noise pattern of $50 \%-50 \%-5 \%$.

\section{FAST EXACT EUCLIDEAN DISTANCE (FEED) MAPS}

The principle of FEED ${ }^{5}$ is that each object pixel feeds its Euclidean Distance (ED) to each pixel in the image. Each pixel then takes the minimum of all received EDs to obtain its distance to the set of object pixels. The naive algorithm then becomes:

(1) initialize $E^{2} D M(p)=$ if $(p \in O)$ then 0 , else $\infty$

(2) foreach $q \in O$

(3) foreach $p$

(4) update : $E^{2} D M(p)=\min \left\{E^{2} D M(p), E D(q, p)\right\}$

where $O$ is the set of object pixels.

Several speedup methods are applied to this naive algorithm. In line (2), only the "border" pixels $B$ of $O$ have to be considered because the minimal $E D$ from any background pixel to the set $O$ is the distance from that background pixel to a border pixel $B$ of $O$. A border pixel $B$ is defined here as an object pixel with at least one of its four four-connected pixels in the background. The $E D$ in line (4) can be retrieved from a pre-computed matrix. Moreover, the number of pixels that have to be considered in line (3) can be limited to only those that have an equal or smaller distance to the current $B$ than to any object pixel $q$.

In principle, when all $q$ are taken into account, each background pixel needs to be updated only once. However, then the search for the $q$ and the bookkeeping of which pixels to update for the current $B$ takes much more time than the time gained by not updating more than once. Therefore, the considered $q$ 's are limited to neighbors of $B$ because they provide the largest reductions in the number of pixels to update and to $q$ on certain radial lines starting at $B$, because they have a simple and fast bookkeeping scheme ${ }^{5,11}$. This results in FEED implementations, which are only a factor one to two slower than chamfer distance transformations ${ }^{9,12}$ and a factor two or more faster than semi-exact ED transformations ${ }^{10,13}$, as was shown for $2 \mathrm{D}^{5}$, for $3 \mathrm{D}^{14}$, and for images that incorporate multiple classes (of objects) ${ }^{11}$.

As described in Section 1, for a sequence of images with stationary and moving objects the $\mathrm{E}^{2} \mathrm{DMs}$ of the sequence can be obtained very fast by combining the (fixed) $\mathrm{E}^{2} \mathrm{DM}$ of the stationary objects with the (changing) $\mathrm{E}^{2} \mathrm{DM}$ for the moving objects. This is illustrated in Figure 2, where (a) shows the $\mathrm{E}^{2} \mathrm{DM}$ of the stationary pixels of Figure 1 (a) and (b) shows the area that has to be updated to add the influence of a moving object.

We have shown ${ }^{4}$ that the $\mathrm{E}^{2} \mathrm{DMs}$ of a sequence of more than a few images can be obtained faster than city-block $^{8}$ or 3,4 chamfer $^{9}$ distance maps. Compared to the original implementation, some additional speedup 


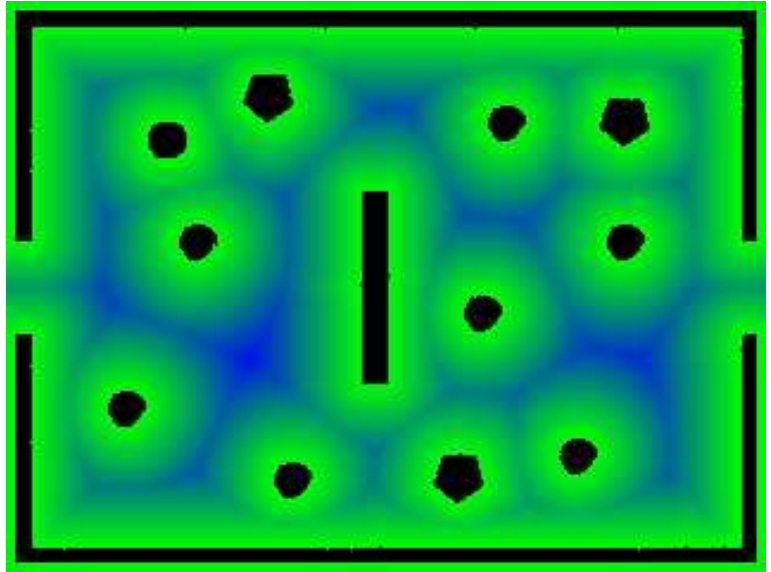

(a)

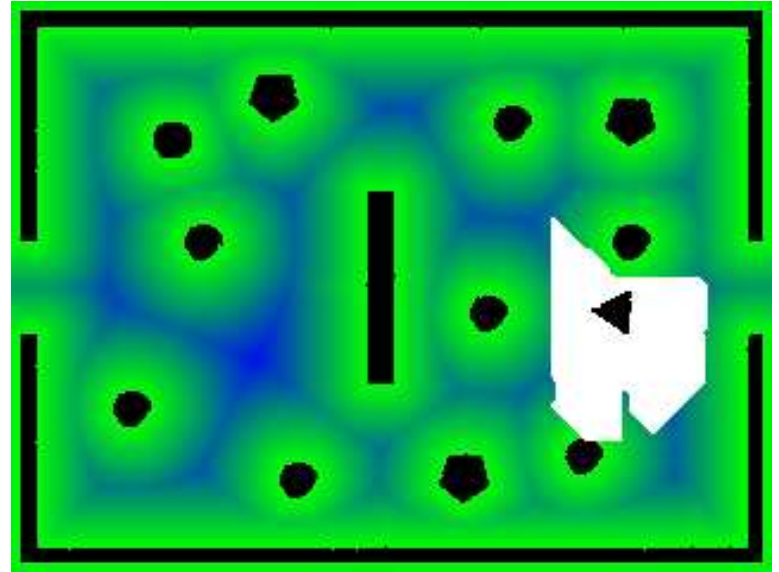

(b)

Figure 2. Two Euclidean Distance Maps ( $\left.E^{2} D M s\right)$ : (a) The $E^{2} D M$ of the stationary pixels of Figure 1 (a). A color range from bright green to bright blue is used here for EDs ranging from 0 to the maximum ED in the images. Image (b) shows in white the area that was updated for adding the Euclidean Distances to the triangular moving object.

methods have been applied. The border pixels of the stationary objects are determined and stored in two lists, one for the $x$ and one for the $y$ direction. This speeds up the search for object pixels $q$ in the horizontal and vertical directions for each border pixel. Further, the influence of the maximum distance $d_{\max }$ in the stationary $\mathrm{E}^{2} \mathrm{DM}$ was better taken into account. It defines a maximum to the distance that a border pixel of a moving object might have to feed its ED to. This circle was in the original implementation approximated by its outside square. In the current implementation, it is approximated by its outside octagon. In total, these two additions provide an additional speedup of 10 to $20 \%$, compared to the original implementation.

\section{REAL-TIME AND EXACT MOTION DETECTION}

The processing chain for the real-time and exact motion detection, shown in Figure 3, consists of two phases. In the initialization phase, a number of sequential images are used to determine the pixels of the stationary objects. This is followed by a number of pre-calculations to speedup the processing of the following frames. In addition, pre-calculations are done for the generation of images to display the results of the processing. How the results of the processing chain are used depends on the specific application the program is used for; for this paper, the results were simply visualized.

In the processing phase, for each next frame the object pixels in it are classified as stationary or moving object pixels, while removing most of the noise pixels. It is verified whether or not the stationary pixels have changed substantially; if they have done so, the initialization phase is entered again. Otherwise, the moving pixels are grouped into objects in a robust way and the remaining noise pixels, which give small noise objects, are removed. Then, distances from each moving object to the stationary and to the other moving objects are calculated. From that point on, they can be handled in an application-specific way. In case an object with processing capabilities is present (for the purposes of this paper we simply assume that one of the objects has this capability), its $\mathrm{E}^{2} \mathrm{DM}$ to the stationary and to the other moving objects is calculated. Next, this $\mathrm{E}^{2} \mathrm{DM}$ can be communicated to the intelligent object, depending on the particular circumstances. Finally, the displays for the visualization of the results are generated.

In Table 1, the execution time for each program block is given for the image sequence with the large frames and the most noise on both the execution platforms. The reported times show a clear difference between the two execution platforms. The AMD platform is faster in the generation of display images; for the other program parts, the INTEL platform is faster. Please note that the reported times do not include the time needed for grabbing of frames for a particular application nor for processing the frames to obtain the binary frames needed 


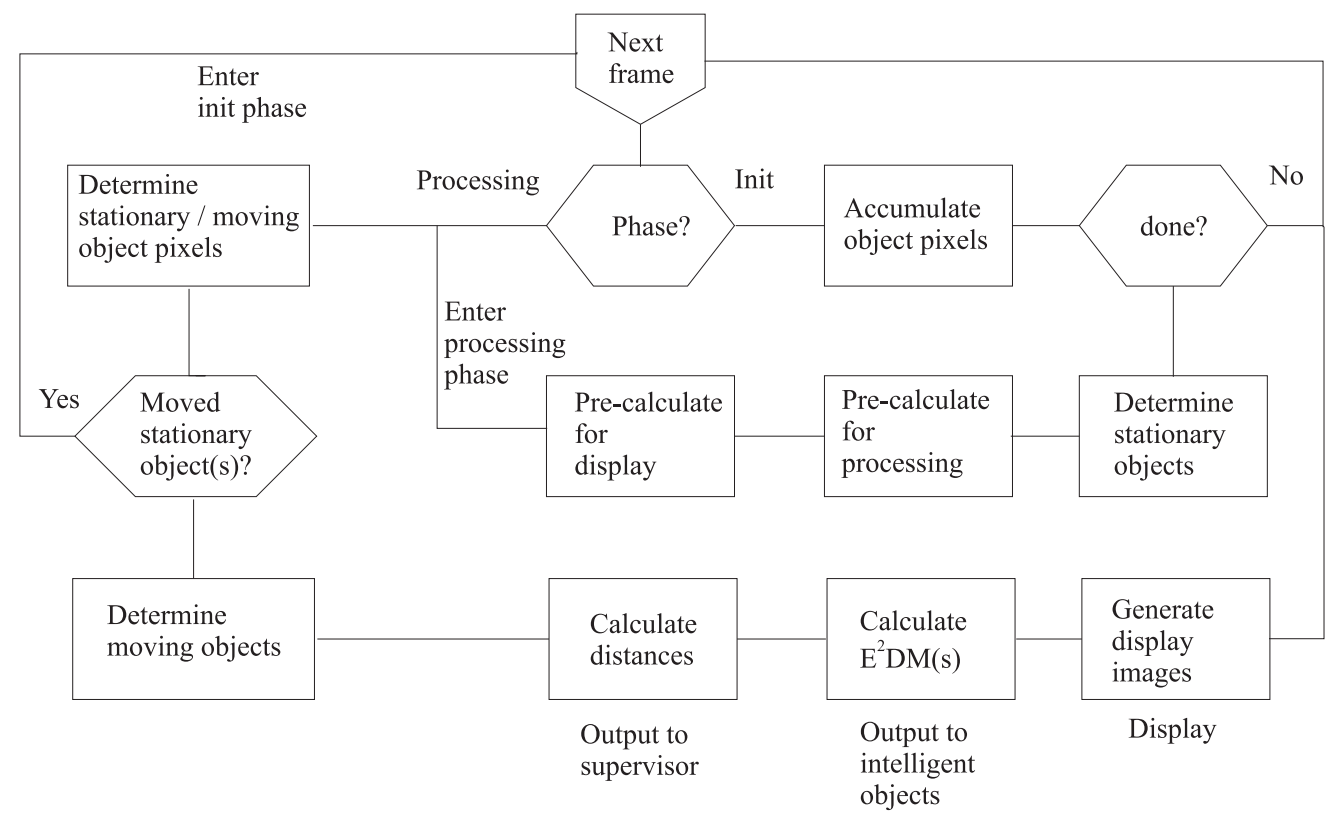

Figure 3. The processing chain for real-time and exact motion detection, as presented in this paper.

for our processing chain. However, the measured times are small compared to the inter frame times of typical webcam like cameras, which are in the order of 50 to 80 milliseconds.

The following design guidelines were used to obtain the fast execution times:

1. Pre-calculation of information: For frame-to-frame processing, information is used that only depends on the information obtained in the initialization phase. Pre-calculating this information and storing it in suitable data structures allows faster processing in the processing phase. An example is the position information of borders of the stationary objects stored in two lists, mentioned in Section 3.

2. Restriction of data movement: Often in the processing phase an image or matrix is initialized with precalculated information and then information derived from the current frame is added. By keeping track of which parts(s) of the data structure has been changed, only those parts have to be reinitialized for the next frame. An example is the situation shown in Figure 2, a bounding box of the changed, white area in image (b) is determined and used in the next frame to restore the original situation as shown in image (a).

3. Combination of loops and test: Often program parts with logically distinct purposes use nearly the same loops and test structures, they can be combined to avoid double execution of the common parts. An example is the detection of the moving objects combined with the detection of moved stationary objects. The opposite of this can also sometimes be exploited: the removal of noise is distributed over a number of other program parts to get a free ride on tests performed for those parts.

\section{DETAILS OF THE PROCESSING CHAIN}

\subsection{Determining stationary pixels}

For a number of frames, currently set to five, the number of occurrences of an object pixel are counted for each pixel location in a count matrix. Here, an object pixel is only counted if at least one of its four-connected neighbors is also an object pixel. For being considered as a stationary object pixel, a location must have received five counts or three or four counts, but then all its four-connected neighbors must have received five counts. Further, holes of one background pixel surrounded by 4 stationary pixels are filled. This procedure is fast and sufficient even for sequences with a large amount of noise. 
Table 1. Execution times for the program blocks in the processing chain. They are given here for the sequence of frames with size $640 \times 480$ and the most noise (50\%-50\%-5\% pattern), on both execution platforms.

\begin{tabular}{llrr}
\hline Phase & Program block & Time AMD & Time INTEL \\
\hline Initialization & Accumulate objects pixels for 5 & $9.7 \mathrm{~ms}$ & $7.7 \mathrm{~ms}$ \\
& frames & & \\
(time per 5 frames) & Determine stationary objects & $2.5 \mathrm{~ms}$ & $2.0 \mathrm{~ms}$ \\
& Pre-calculate for further processing & $34.4 \mathrm{~ms}$ & $18.5 \mathrm{~ms}$ \\
& Pre-calculate for generation of display & $10.3 \mathrm{~ms}$ & $14.5 \mathrm{~ms}$ \\
& images & & \\
Processing & Determine stationary/ moving object & $2.6 \mathrm{~ms}$ & $2.0 \mathrm{~ms}$ \\
& pixels & & \\
(time per frame) & Determine moving objects & $0.7 \mathrm{~ms}$ & $0.3 \mathrm{~ms}$ \\
& Calculate distances & $1.2 \mathrm{~ms}$ & $0.3 \mathrm{~ms}$ \\
& Calculate E DM(s) & $1.4 \mathrm{~ms}$ & $2.0 \mathrm{~ms}$ \\
\hline
\end{tabular}

Please note that for the determination of stationary pixels, the moving objects have to move sufficiently fast: in the used number of frames, there should be at least two frames in which the pixels of the moving object do not overlap. If there is an overlap, some part of the moving object will be considered as stationary object pixels. When that part is large enough, the processing phase will detect for one of the following frames a substantially change of that part and then reenter the initialization phase. If this happens too often, our program keeps functioning but too few frames are used for deriving video surveillance values.

A simple strategy to detect whether or not moving object move too slow is to track the interval between initializations. Then, a first counter measure would be to adapt the number of frames used for initialization to the determined intervals. A next step would be to switch to a more elaborate statistical processing of the pixels in the initialization frames, like giving weights to the counts, depending on neighboring pixels.

\subsection{Pre-calculation}

In Table 2, the most important pre-calculations are given with their execution times. Here, we will provide some additional information. The input matrix for each next frame is initialized with the stationary object pixels to which then the object pixels in the frame are added.

In the pixel classification matrix, each pixel location is classified as being: (a) inside a stationary object, when the location and its four-connected neighbors are occupied by stationary object pixels; (b) outside the stationary objects, when the location and its four-connected neighbors are occupied by background pixels; (c) otherwise the location is in the border area. This matrix is used in the first processing block to speed up the processing phase of that block.

Table 2. A list of the operations performed as pre-calculation and the execution time for the sequence of frames with size $640 \times 480$, with the most noise (50\%-50\%-5\% pattern), on the AMD system.

\begin{tabular}{lr}
\hline Pre-calculation operation & Time \\
\hline Initialization matrix for the input matrix for each next frame & $0.5 \mathrm{~ms}$ \\
Calculation of E ${ }^{2} \mathrm{DM}_{\text {stationary }}$ & $24.8 \mathrm{~ms}$ \\
Determination of the borders of the stationary objects & $1.3 \mathrm{~ms}$ \\
Initialization of E ${ }^{2} \mathrm{DM}_{\text {stationary+moving }}$, maximum distance & $4.1 \mathrm{~ms}$ \\
Calculation of the pixel classification matrix & $2.8 \mathrm{~ms}$ \\
Calculation and initialization of display of moving objects & $1.7 \mathrm{~ms}$ \\
Calculation and initialization of display of E $\mathrm{DM}_{\text {stationary+moving }}$ & $8.6 \mathrm{~ms}$ \\
\hline
\end{tabular}




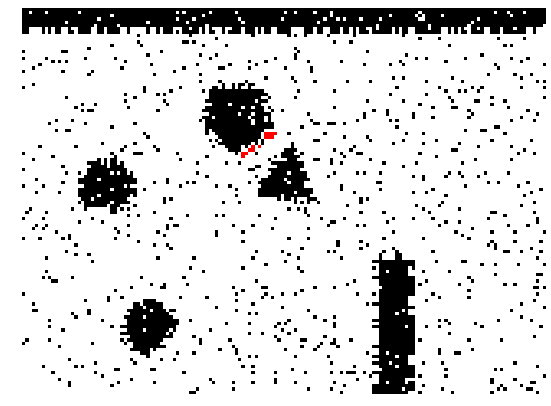

(a)

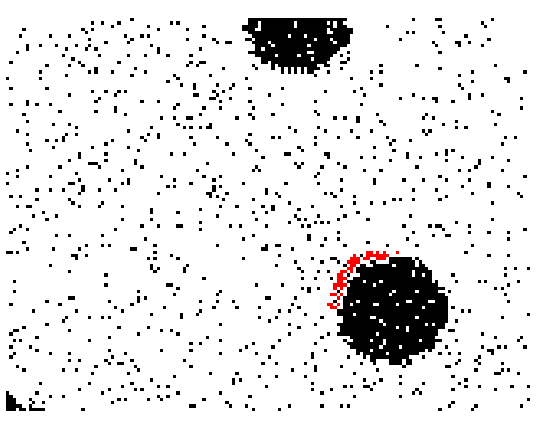

(b)

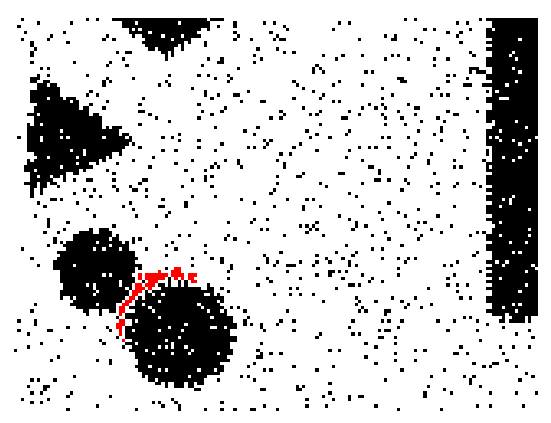

(c)

Figure 4. Detection of movement of stationary objects, where a red pixel indicates a position where a stationary object pixel is replaced in the current frame with a background pixel. (a) shows part of the image in the sequence with $320 \times 240$ frames after a stationary object is displaced by a moving object. (b) and (c) show part of the image in the sequence of $640 \times 480$ frames, where a stationary object moves spontaneously, respectively is displaced by a moving object.

The determination of the borders of the stationary objects and the filling of the pixel classification matrix

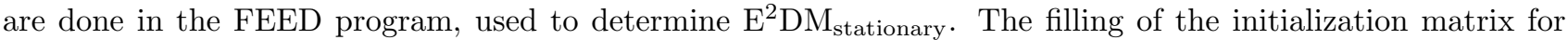
$\mathrm{E}^{2} \mathrm{DM}_{\text {stationary+moving }}$ is combined with the calculation of the maximum distance in $\mathrm{E}^{2} \mathrm{DM}_{\text {stationary }}$.

\subsection{Determine stationary / moving object pixels}

The input matrix for each new frame is initialized with the stationary input pixels. As described in the previous Section, we keep track of which parts of it were changed for the previous frame and only those parts are reinitialized. Then a single scan over the frame is performed both to detect moved stationary objects and to determine the moving object pixels.

Each background pixel is checked for being located inside a stationary object, using the pixel classification matrix described in the previous subsection. If that is the case and all its four-connected pixels are also background pixels, the pixel is counted as a changed stationary object pixel. Figure 4 shows the detected changed stationary object pixels for the three occurrences in the video sequences of a moved stationary object for the sequences with the most noise.

A simple threshold on the number of changed stationary object pixels is sufficient to define a substantial movement of a stationary object, in which case the program chain reenters the initialization phase. In the images shown in Figure 4, the number of changed pixels is 22, 54, and 73, while in the other images of the sequences the maximal number of changed pixels is two. For the images with the least noise the number of changed pixels is 36,99 , and 92 , while in the other images no changed pixels are detected. Thus, our movement detector is very robust against noise and is able to detect small changes.

In the same single scan over the frame, each object pixel is checked for being located outside the stationary objects and for being four-connected to at least two other object pixels. If the latter is the case, the pixel is considered to be a moving object pixel, indicated by a value of 127 in the input matrix. Then, a check is made for small holes in the moving object by determining whether the pixels at the same column in the previous two scan lines form such a hole. By changing the pixels in the hole to moving object pixels, the hole is removed. This procedure removes all of the single pixel holes and about half (the vertical ones) of the double pixel holes in the moving objects. Further, during the scan, a list is generated containing the position of the first and last moving object pixel for each scan line. This is used to decrease the execution time of the next processing block.

Figure 5(b) shows the result of the above processing for part of a large frame, which is shown in Figure 5(a). Please note that the holes in the moving object are removed except for the horizontal hole of two pixels. In addition, most of the noise object pixels outside the moving objects are removed. The remaining ones are mostly located close to the borders of the fixed objects, caused by the large amount of noise present in the borders 


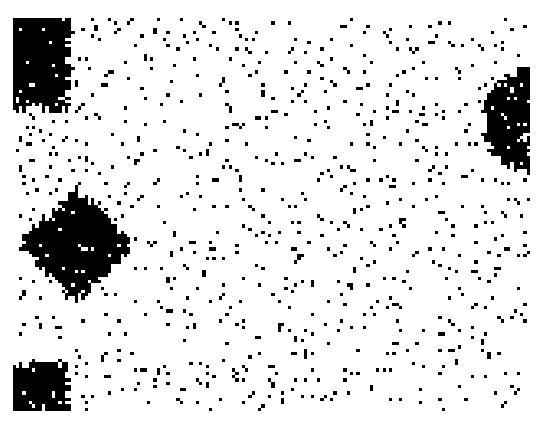

(a)

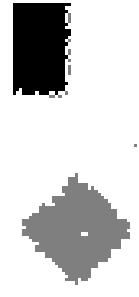

(b)

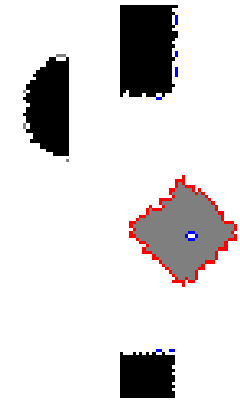

(c)

Figure 5. Locating the moving objects: (a) shows part of a large input frame. (b) shows the detected moving object pixels indicated in gray. (c) shows (in color) the borders of the detected objects; note that a hole in an object also generates an object.

\subsection{Determine moving objects}

The determination of the moving objects is performed in three steps: 1) locating the border pixels of the objects, 2) finding the objects, and 3) removing the noise objects.

For locating the border pixels of the moving objects, a single scan over the frame is made, restricting the number of pixels to be considered using the list described in the previous Section. Isolated moving objects pixels, defined as having no four-connected moving objects pixels, are detected and removed during this scan. The locations of the border pixels found are stored in a list in order to speed up further processing.

The moving objects are found by identifying their contours consisting of their eight-connected border pixels. These contours are constructed using a variant of a standard "blob coloring" algorithm. This variant operates directly on the list of border pixels using a single forward scan over the list. It uses a direct backward scan to resolve each color (the contour number) equivalence. The assigned contour number of each border pixel is stored in a separate list. Each object has one outside contour and one inside contour for each hole in the object. For the purposes of this paper, an inside contour is considered to be the outside contour of a hole object.

For the removal of noise objects, hole objects have to be distinguished from the other objects. This is done in a simple and very fast way by counting the number of background and the number of moving object pixels between consecutive pairs of its contour pixels on each scan line. If there are more background than moving object pixels counted this way, the object is considered to be a hole. There might be rather pathological situations in which the above test fails. As this does not happen with our input sets, we have not implemented more complicated tests (for which many possibilities exists), to cure this potential problem. As hole objects have at least four contour pixels, the above test is not performed for objects with less than four contour pixels, which constitute close to $90 \%$ of the total number of noise objects.

The non-hole noise objects can only be distinguished from the real objects, based on their size, when considering each frame separately. The maximum occurring number of pixels on the contour of noise objects is nine in all the ten video sequences. For comparison, the minimal occurring contour size of the real objects is 42 in the small frames and 67 in the large frames. Thus, smaller objects than used in our input sets can be distinguished from noise objects, using a simple threshold on the contour size. Switching to a more complicated size measure, like the number of inside pixels, will decrease the size of real objects that can be distinguished from noise.

Figure 5(c) shows the result of the object detection for part of a large frame, which is shown in Figure 5(a). In the full frame there were 775 border pixels of moving pixels determined from which 182 objects were detected. This was reduced to the three real objects by the removal of the noise objects.

\subsection{Distance calculation}

The minimal distance from each moving object to the stationary objects is calculated by taking the minimum of the distances in the $E^{2} D M_{\text {stationary }}$ at the locations of all the contour points of the moving object. This is 


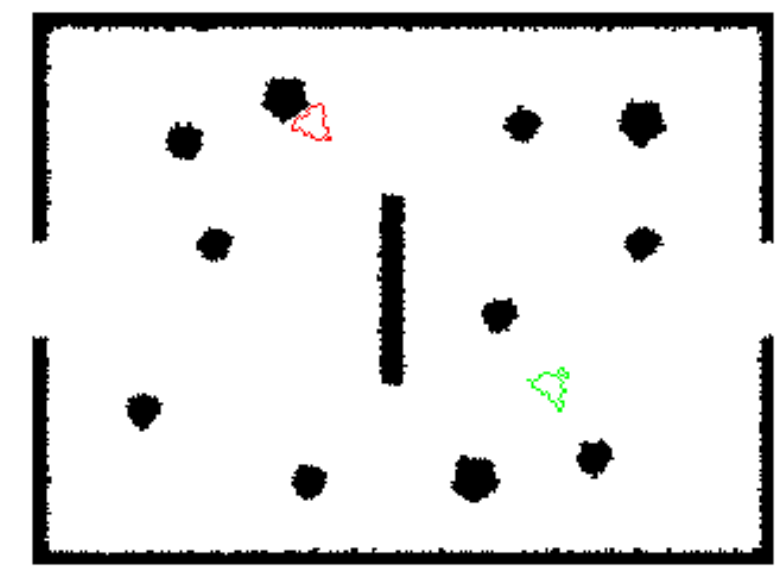

(a)

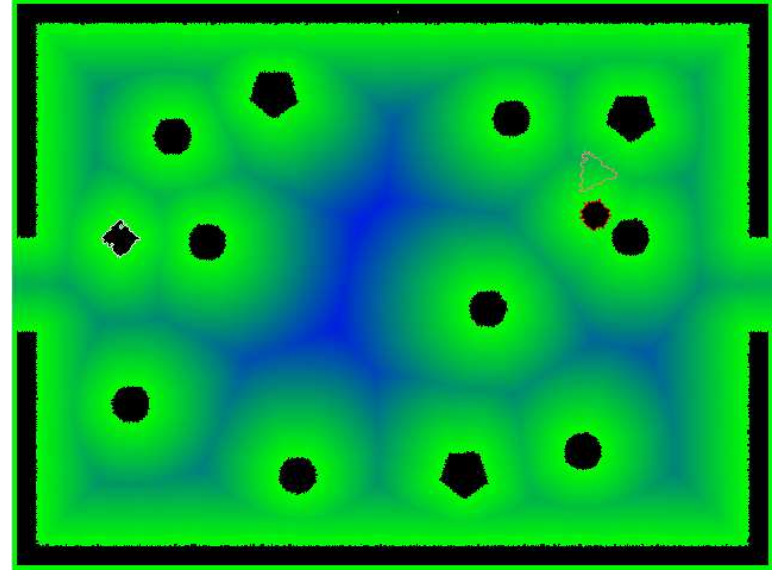

(b)

Figure 6. Supervisor displays: (a) The pixels of the stationary objects and the contours of the moving objects are shown. The color of the latter encodes the determined distances; e.g., red indicates to close to another object. (b) The $\mathrm{E}^{2} \mathrm{DM}$ for the triangular moving object of which the contour is shown.

a very fast operation, taking less than 0.02 milliseconds of the 0.4 milliseconds, reported for the total distance calculation in Table 1. The minimal distance from each moving object to other moving objects is obtained by taking the minimum of the distances between all pairs of points on the contours of the objects. This operation takes most of the time for this processing block.

A frame-to-frame tracking of objects is implemented which uses as track measure the sum of the absolute value of the difference between the $x$ position of the objects, the $y$ position, and the number of contour pixels. Here, the positions are taken as the average positions of all contour points. This tracking is used to select the same object for the calculation in the next processing block and for showing the supervisor a list of disappeared, new, and continued objects.

\subsection{Calculate $\mathrm{E}^{2} \mathrm{DM}(\mathrm{s})$ and display generation}

The first appearing object in a sequence is chosen and tracked, as described in the previous subsection. The $\mathrm{E}^{2} \mathrm{DM}$ is calculated for the stationary objects and the other moving objects in the frame using the FEED ${ }^{4}$ method, as described in Section 3.

Figure 6 shows examples of the two generated displays. Figure 6 (a) shows the stationary pixels and the contours of the detected moving objects for the frame given in Figure 1 (a). The contours are color coded; e.g., red indicates being to close to another objects, according to some chosen video surveillance parameters. Figure 6 (b) shows the maneuver space for the triangular object in Figure 1 (b) of which only the contour is drawn. This consists of the combined $\mathrm{E}^{2} \mathrm{DM}$ for the other moving objects and for the stationary objects. The contours of all moving objects are also color coded.

\subsection{Overall timing results}

In Table 3, the execution times are provided for the different video sequences and execution platforms (see Section 2). The times for the initialization phase are for the full processing of the frames used for initialization; those for the processing phase are given per frame. For both phases, the times are given separately for the processing to obtain the distances as the video surveillance results and for the processing needed to generate the display images for a human supervisor. The latter is only presented in this paper as an example of what can be done with the produced video surveillance results.

From inspection of the programming code, it can be derived that the execution times for generating the display images should be rather independent from the noise applied to the video sequences. The times should 


\begin{tabular}{|c|c|c|c|c|c|c|c|c|c|}
\hline \multicolumn{2}{|c|}{ Sequence } & \multicolumn{4}{|c|}{ AMD platform } & \multicolumn{4}{|c|}{ INTEL platform } \\
\hline & & \multicolumn{2}{|c|}{ Init phase } & \multicolumn{2}{|c|}{ Per frame } & \multicolumn{2}{|c|}{ Init phase } & \multicolumn{2}{|c|}{ Per frame } \\
\hline Size & Noise & process. & display & process. & display & process. & display & process. & display \\
\hline \multirow[t]{5}{*}{$320 \times 240$} & $10 \%-10 \%-1 \%$ & 9.15 & 2.81 & 0.88 & 0.41 & 5.89 & 3.91 & 0.59 & 0.38 \\
\hline & $20 \%-20 \%-2 \%$ & 9.59 & 2.88 & 0.91 & 0.40 & 6.42 & 3.90 & 0.61 & 0.38 \\
\hline & $30 \%-30 \%-3 \%$ & 10.15 & 2.77 & 0.94 & 0.41 & 7.02 & 3.85 & 0.65 & 0.38 \\
\hline & $40 \%-40 \%-4 \%$ & 10.42 & 2.80 & 1.02 & 0.42 & 7.39 & 3.85 & 0.70 & 0.40 \\
\hline & $50 \%-50 \%-5 \%$ & 10.62 & 2.83 & 1.13 & 0.42 & 7.66 & 3.91 & 0.73 & 0.40 \\
\hline \multirow[t]{5}{*}{$640 \times 480$} & $10 \%-10 \%-1 \%$ & 39.88 & 10.63 & 4.12 & 1.37 & 24.03 & 14.67 & 2.49 & 1.87 \\
\hline & $20 \%-20 \%-2 \%$ & 42.66 & 10.30 & 4.20 & 1.42 & 25.63 & 14.38 & 2.57 & 1.91 \\
\hline & $30 \%-30 \%-3 \%$ & 45.41 & 10.27 & 4.32 & 1.44 & 27.00 & 14.57 & 2.72 & 1.95 \\
\hline & $40 \%-40 \%-4 \%$ & 46.78 & 10.56 & 4.52 & 1.42 & 27.98 & 14.42 & 2.90 & 1.93 \\
\hline & $50 \%-50 \%-5 \%$ & 46.60 & 10.33 & 4.94 & 1.40 & 28.32 & 14.54 & 3.21 & 1.99 \\
\hline
\end{tabular}

Table 3. Timing results in $\mathrm{ms}$ for all ten sequences on the two execution platforms. Times are given separately for the two phases of our processing chain. Further, times are given separately for the core processing (process.) that produces the video surveillance results and for a visual display of them (display), which is just given as an example of what can be done with the results.

be constant in the initialization phase (only dependent on the frame size), while in the processing phase they should show a small variation of a few percent. The measured root mean square deviation for the time series varies from 0.8 to $1.6 \%$ in the initialization phase and from 1.9 to $2.6 \%$ in the processing phase. This confirms the stated accuracy of $2 \%$ for our time mesurement (see Section 2).

The reported times also show a difference between the two execution platforms. For the generation of display images the AMD platform is up to $40 \%$ faster (with exception of the small frames in the processing phase); for the other program parts, the INTEL platform is up to $68 \%$ faster.

\section{DISCUSSION}

We have presented a real-time, accurate, and robust system for automatic distance and motion detection in video sequences. Stationary and moving objects are detected in a robust way, from video sequences taken by a single camera providing a top view of a scene. To obtain fully controlled test environments, an artificial development center for robot navigation is introduced in which several parameters can be set; e.g., number of objects, trajectories and type and amount of noise. Ten different noisy video sequences were generated in order to test both the robustness and the speed of our system. Distances between moving and stationary objects are obtained in a fast and accurate way, using the Fast Exact Euclidean Distance (FEED) $)^{4,5,11,14}$ transform. Each object with processing capabilities (e.g., a robot) is provided with its Exact Euclidean Distance Map ( $\left.\mathrm{E}^{2} \mathrm{DM}\right)$ to the stationary and other moving objects and, for example, can be used to detect and prevent unwanted or possible dangerous situations.

Several restrictions on the content of the video sequences have been identified in this paper and ways to remove them have been discussed. They include the minimum speed of moving objects during the determination of the pixels of the stationary objects (see Section 5.1), the minimal amount of detectable movement of stationary objects (see Section 5.3), and the minimal size of the moving objects in order to be able to separate them from noise objects (see Section 5.4). Further reduction of these minimal values will be one of the topics in our further research. More in general, the fundamental research toward the application of $\mathrm{E}^{2} \mathrm{DMs}$ in various controlled environments will continue. In addition, parallel motion detection, in line with Schouten et al. ${ }^{4}$, will be employed for various types of objects, each with their own characteristics, as was done for images in Van den Broek et al. ${ }^{11}$. For the latter purpose, the artificial development center for robot navigation will be extended. This will continue until environments can be created that almost touch reality.

In parallel with the controlled tasks, the application of motion detection in real life recordings will be conducted. Without any doubt, these recordings will give birth to a range of problems that were not encountered, so far. One of the most striking is occlusion. In contrast with video surveillance techniques, humans are able 
to track moving objects with a striking ease, even when temporarily occluded. However, what principles lay behind this capability is far from clear. Only in the last five years, attempts have been made to extend occlusion research to the domain of video surveillance ${ }^{1}$. Hence, the problem of occlusion forms one of the big challenges for the application of $\mathrm{E}^{2} \mathrm{DMs}$ on video surveillance.

With the increase of attention for safety issues in modern societies, the need for automated, preferably, autonomous, real-time video surveillance techniques is pressing. The current paper uses distance maps for realtime, exact tracking of moving objects, which is an unique approach. Moreover, the processing chain presented is robust to noise. This line of research will be continued and, in parallel, will be extended to real life video surveillance. Considering the promising results, the processing chain presented can be expected to become of significant importance for video surveillance.

\section{REFERENCES}

1. A. Hampapur, L. M. Brown, J. Connell, M. Lu, H. Merkl, S. Pankanti, A. W. Senior, C.-F. Shu, and Y.-L. Tian, "Multi-scale tracking for smart video surveillance," IEEE Transactions on Signal Processing 22(2), pp. 38-51, 2005.

2. A. R. Dick and M. J. Brooks, "Issues in automated visual surveillance," in Proceedings of the International Conference on Digital Image Computing: Techniques and Applications (DICTA 2003), pp. 195-204, (Sydney, Australia), December 2003.

3. A. Amer and C. Regazzoni, "Introduction to the special issue on video object processing for surveillance applications," Real-Time Imaging 11(3), pp. 167-171, 2005.

4. Th. E. Schouten, H. C. Kuppens, and E. L. van den Broek, "Timed Fast Exact Euclidean Distance (tFEED) maps," Proceedings of SPIE (Real Time Imaging IX) 5671, pp. 52-63, 2005.

5. Th. E. Schouten and E. L. van den Broek, "Fast Exact Euclidean Distance (FEED) Transformation," in Proceedings of the 17th IEEE International Conference on Pattern Recognition (ICPR 2004), J. Kittler, M. Petrou, and M. Nixon, eds., 3, pp. 594-597, (Cambridge, United Kingdom), 2004.

6. A. Hajdu and L. Hajdu, "Approximating the Euclidean distance using non-periodic neighbourhood sequences," Discrete Mathematics 283(1-3), pp. 101-111, 2004.

7. C. Fouard and G. Malandain, "3-D chamfer distances and norms in anisotropic grids," Image and Vision Computing 23(2), pp. 143-158, 2005.

8. A. Rosenfeld and J. L. Pfaltz, "Distance functions on digital pictures," Pattern Recognition 1, pp. 33-61, 1968.

9. G. Borgefors, "Distance transformations in digital images," Computer Vision, Graphics, and Image Processing: An International Journal 34, pp. 344-371, 1986.

10. F. Y. Shih and Y.-T. Wu, "Three-dimensional Euclidean distance transformation and its application to shortest path planning," Pattern Recognition 37(1), pp. 79-92, 2004.

11. E. L. van den Broek, Th. E. Schouten, P. M. F. Kisters, and H. C. Kuppens, "Weighted Distance Mapping (WDM)," in Proceedings of the IEE International Conference on Visual Information Engineering (VIE2005), N. Canagarajah, A. Chalmers, F. Deravi, S. Gibson, P. Hobson, M. Mirmehdi, and S. Marshall, eds., pp. 157164, Wrightsons - Earls Barton, Northants, Great Britain, (Glasgow, United Kingdom), 2005.

12. S. Svensson and G. Sanniti di Baja, "Using distance transforms to decompose 3d discrete objects," Image and Vision Computing 20(8), pp. 529-540, 2002.

13. F. Y. Shih and Y.-T. Wu, "Fast euclidean distance transformation in two scans using a $3 \times 3$ neighborhood," Computer Vision and Image Understanding 93(2), pp. 195-205, 2004.

14. Th. E. Schouten, H. C. Kuppens, and E. L. van den Broek, "Three dimensional fast exact euclidean distance (3D-FEED) maps," Proceedings of SPIE (Vision Geometry XIV) 6066, p. [in press], 2006. 